far, no true resting sporangia have been observed in either agar or water cultures.

The present species was first found by Karling ${ }^{8}$ as a contaminant growing in the trichomes of corn while he was working with the physoderma disease. It was described as a weak parasite and saprophyte in trichomes and parenchyma cells of corn and in cooked internodes of Chara and Nitella. The origin of this fungus was, however, uncertain. In view of the present investigation that it can be readily isolated from an agar plate exposed for airborne organisms, its occurrence in the air seems highly possible.

I wish to acknowledge my appreciation to Prof. S. M. Pady for his advice and helpful suggestions.

This communication was written while a student at the Department of Botany, McGill University, Montreal.

Department of Plant Pathology,

WAN-NIEN SIANG

Washington State College, Pullman,

Weashington. Nov. 3.

1 Pady, S. M., Kelley, C. D., and Polunin, N., Nature, 162, 379 (1948).

' Luckiesh, M., Taylor, A. H., and Holladay, L. I., J. Bact., 52, 55 (1946).

s Karling, J. S., Amer. J. Bot., 26, 512 (1939).

- Couch, J. N., Amer. J. Bot., 28, 704 (1941).

\section{Enhancement by 'Visible' Light of Recovery from Ultra-Violet Irradiation in Animal Cells}

DuLBECCO ${ }^{1}$ has reported that exposure to visible light in the presence of bacterial cells enhances reactivation of coli-bacteriophage which has been inactivated by ultra-violet radiation. Kelner ${ }^{2}$ reported earlier this year that visible light enhances the recovery of conidia of Streptomyces griseus which have been treated with ultra-violet radiation. Some years ago, Whitaker ${ }^{3}$ found what may have been the same kind of effect in the eggs of the alga, Fucus furcatus.

The present note is to report a similar finding in echinoderm eggs, the first case involving animal cells, and thus extending widely the occurrence of this phenomenon. Short doses of ultra-violet radiation (wave-length less than $3200 \mathrm{~A}$.) cause the delay of subsequent cleavages of the eggs of the sea urchin Arbacia punctulata. Gradual recovery occurs, until after a few cleavages the normal cleavage-rate is regained. The return to normal rate is greatly accelerated by light from the short end of the visible spectrum (wave-lengths of approximately 4000$5000 \mathrm{~A}$. as determined in preliminary studies).

Since the effect is so widespread, it is cogent to ask whether some ubiquitous substance is the light absorber in all these cases, and this idea is supported by the general agreement between the range of the action spectrum found by ourselves and that found by Dulbecco in an unrelated organism. The spectral region suggests a carotinoid or possibly riboflavin as the light absorber; these substances are widely distributed and photoactive. It seems unlikely that echinochrome, the characteristic pigment of these eggs, is the light absorber for this effect. because of its spectral absorption. Studies of the action spectrum in various other organisms are desirable.

Attempts to demonstrate this effect in human skin have failed thus far. Skin receiving an erythemic threshold dose of ultra-violet radiation from an artificial source was afterwards exposed to sunlight from which the wave-lengths producing erythema were removed by window glass. The doses of the latter were far in excess of those which would be received in the course of exposure to a threshold erythemal dose of natural sunlight. The average from experiments on seven persons indicated no effect of visible light on the threshold; but the error of such measurements is high 4 , and the experiments too few to rule out a small effect. If some recovery does occur, these experiments indicate that it is probably too small to have great importance as regards sunburn.

\section{Harold F. Blum \\ GORDON M. LOOS \\ JUDITH P. PRICE \\ J. Courtland Robinson}

National Cancer Institute;

Department of Biology,

Princeton University ; and the

Marine Biological Laboratory,

Woods Hole. July 21.

${ }^{3}$ Dulbecco, R., Nature, 163, 949 (1949).

${ }^{2}$ Kelner, A., Proc. U.S. Nat. Acad. Sci., 35, 73 (1949).

${ }^{3}$ Whitaker, D. M., J. Gen. Physiol., 25, 391 (1942).

- Blum, H. F., and Terus, W. S., Amer. J. Physiol., 146, 107 (1946).

\section{Micro-Anatomy of the Hepatic Circulation}

IN the course of studying the role of circulatory changes in the pathogenesis of centrilobular hepatic lesions ${ }^{1,2}$, we have been attempting by various tech. niques, including the use of neoprene latex and indian ink $^{3}$, to clarify certain controversial points in the normal liver circulation.

(1) The branches of the portal vein and hepatic artery which reach the parenchyma spread out to form an apparently continuous network of vessels resembling large sinusoids, from which smaller sinusoids radiate into the lobules. This network appears to lie near the junction of the parenchyma and portal tracts and, in this sense, may be regarded as periportal. It is most distinct around the larger portal tracts and less clearly defined as the tracts become smaller.

The network continues into the hilar region and is spread irregularly over the subcapsular liver surface, where it receives a richer arterial supply than in the deeper substance. Small branches of the portal vein penetrate to the subcapsular region and can be seen breaking up to form the large sinusoidal vessels making up the network.

(2) The network is supplied by branches of the portal vein in two ways: $(a)$ by short vessels which terminate soon after passing the limits of the portal space, and (b) by offshoots from larger branches leaving the parent ramification. The 'short' vessels (Fig. 1) are more frequent towards the termination of the portal venous branches.

A loose venous and arterial plexus lies in the larger portal spaces and in the hilar region, and sends branches to the periportal network. No anastomosis between veins of different portal tracts has been seen within the liver, other than via the sinusoids.

Branches of the portal vein are accompanied by branches of the hepatic artery which may be multiple. The arteries give off many small branches which 